\title{
LA-UR-16-29414
}

Approved for public release; distribution is unlimited.

Title: $\quad$ Move Towards a Balanced 7-County Ecosystem

Author(s): $\quad$ Clow, Shandra Deann

Intended for: Presentation for various uses.

Issued: 
Disclaimer:

Los Alamos National Laboratory, an affirmative action/equal opportunity employer, is operated by the Los Alamos National Security, LLC for the National Nuclear Security Administration of the U.S. Department of Energy under contract DE-AC52-06NA25396. By approving this article, the publisher recognizes that the U.S. Government retains nonexclusive, royalty-free license to publish or reproduce the published form of this contribution, or to allow others to do so, for U.S. Government purposes. Los Alamos National Laboratory requests that the publisher identify this article as work performed under the auspices of the U.S. Department of Energy. Los Alamos National Laboratory strongly supports academic freedom and a researcher's right to publish; as an institution, however, the Laboratory does not endorse the viewpoint of a publication or guarantee its technical correctness. 


\section{Move Towards a Balanced 7-County Ecosystem}

Attract \& retain technical talent \& Millennials in NNM, either at LANL or within entrepreneurial ecosystem

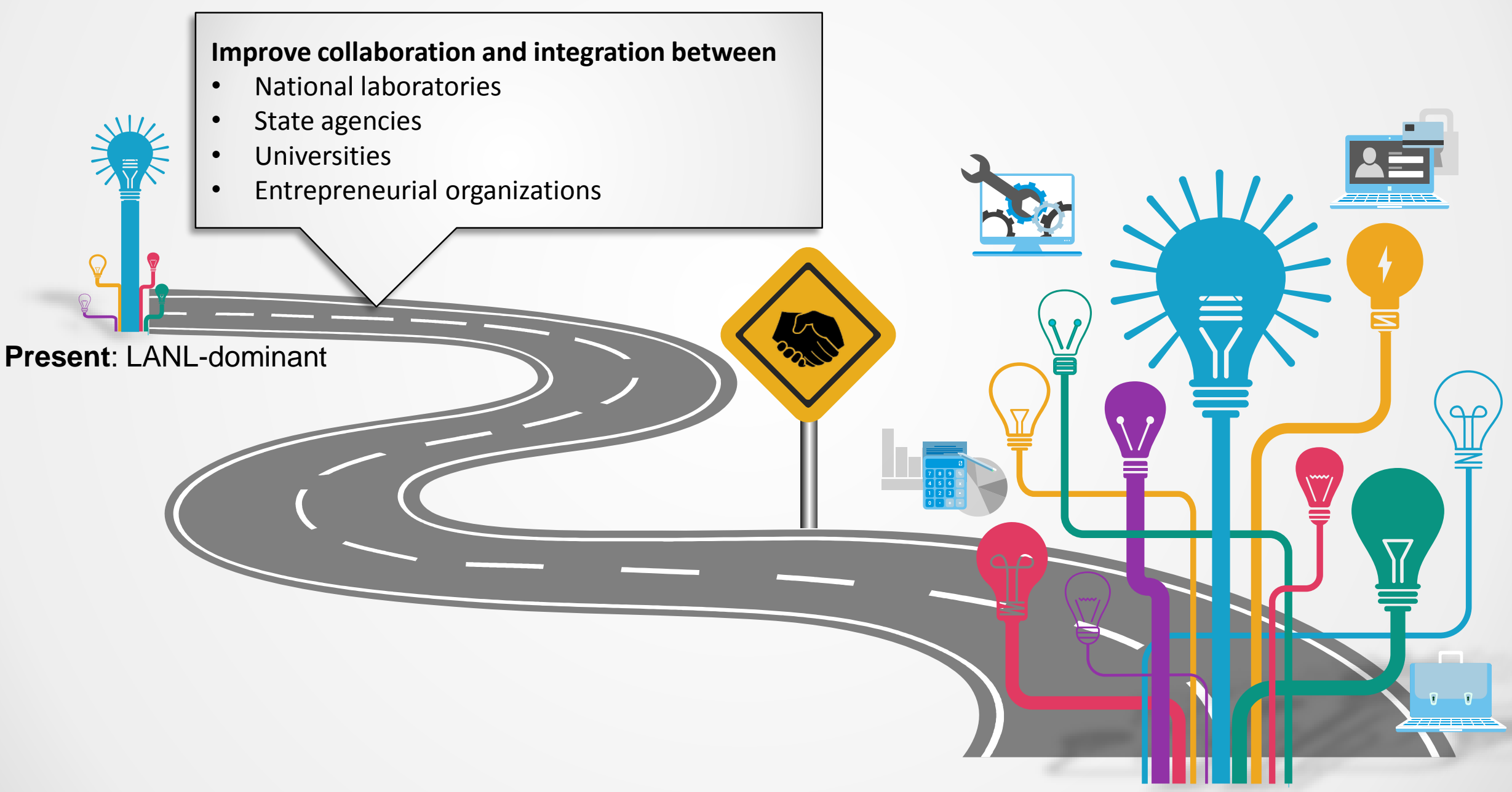

Future: Diversified, Balanced Ecosystem 


\section{The Faces of Entrepreneurship in NNM}

The pursuit of Opportunity beyond the Resources you currently control

-Prof. Howard Stevenson Harvard Business School
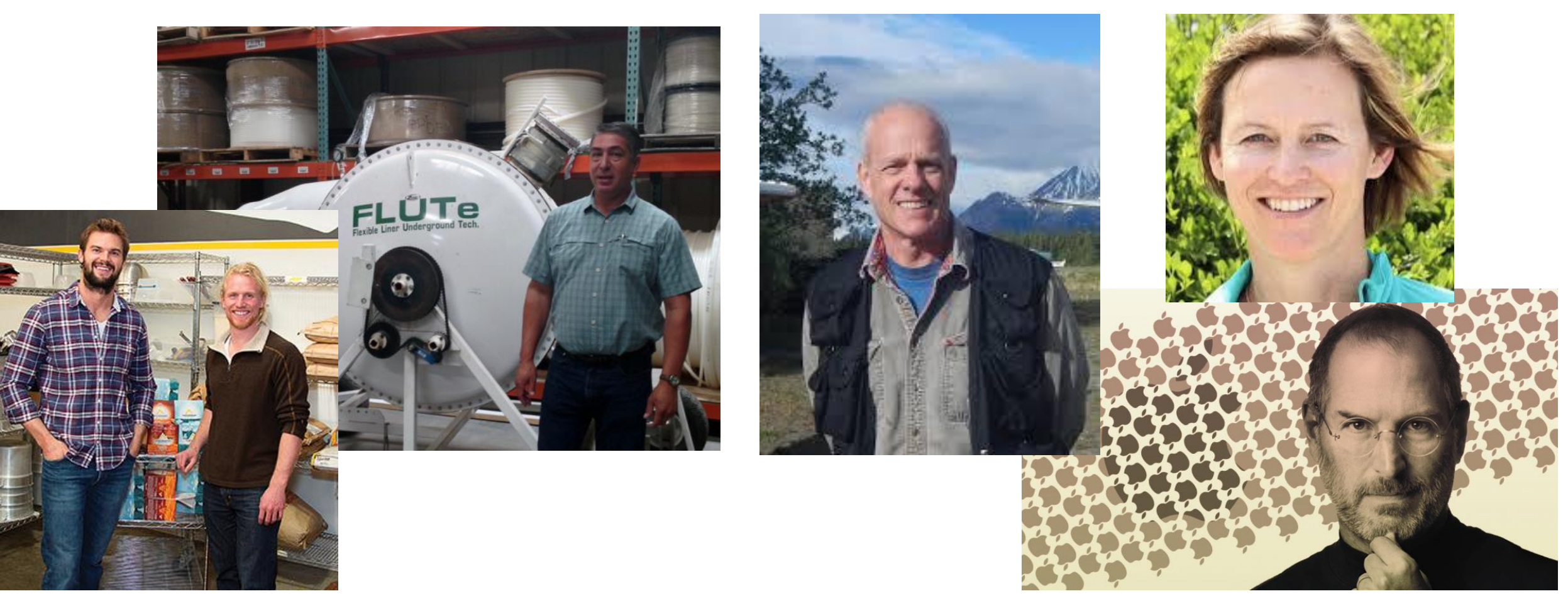


\section{How do we foster growth in small companies in NM?}
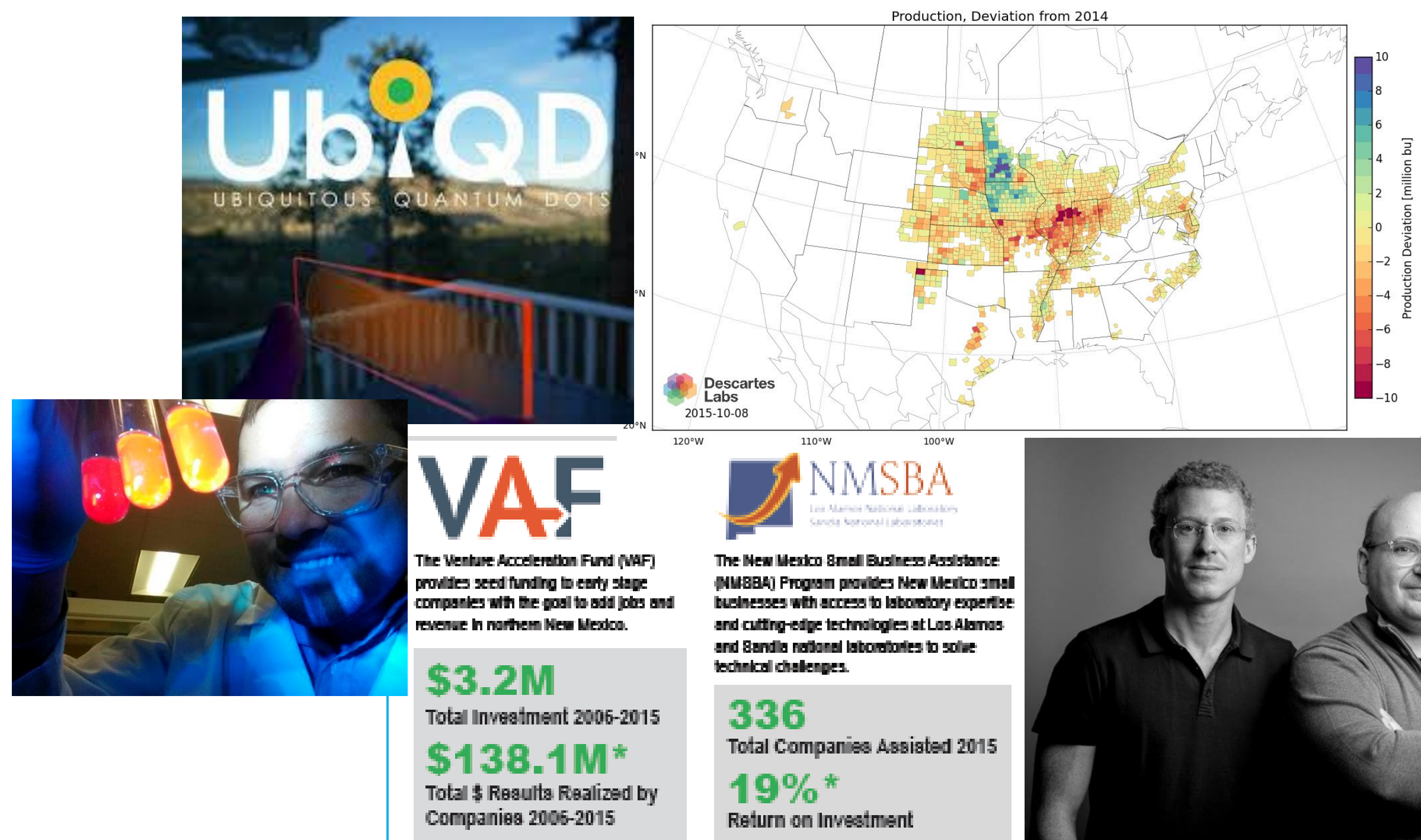

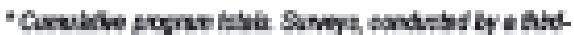

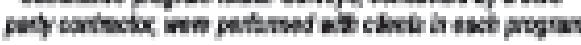

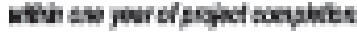

VAF companies grew economically $43 x$ our investment, and NMSBA clients grew $11 \mathrm{x}$ the state's investment. 


\section{Innovation Funnel}

Provide LANL staff opportunities to be more involved in and educated on technology transition

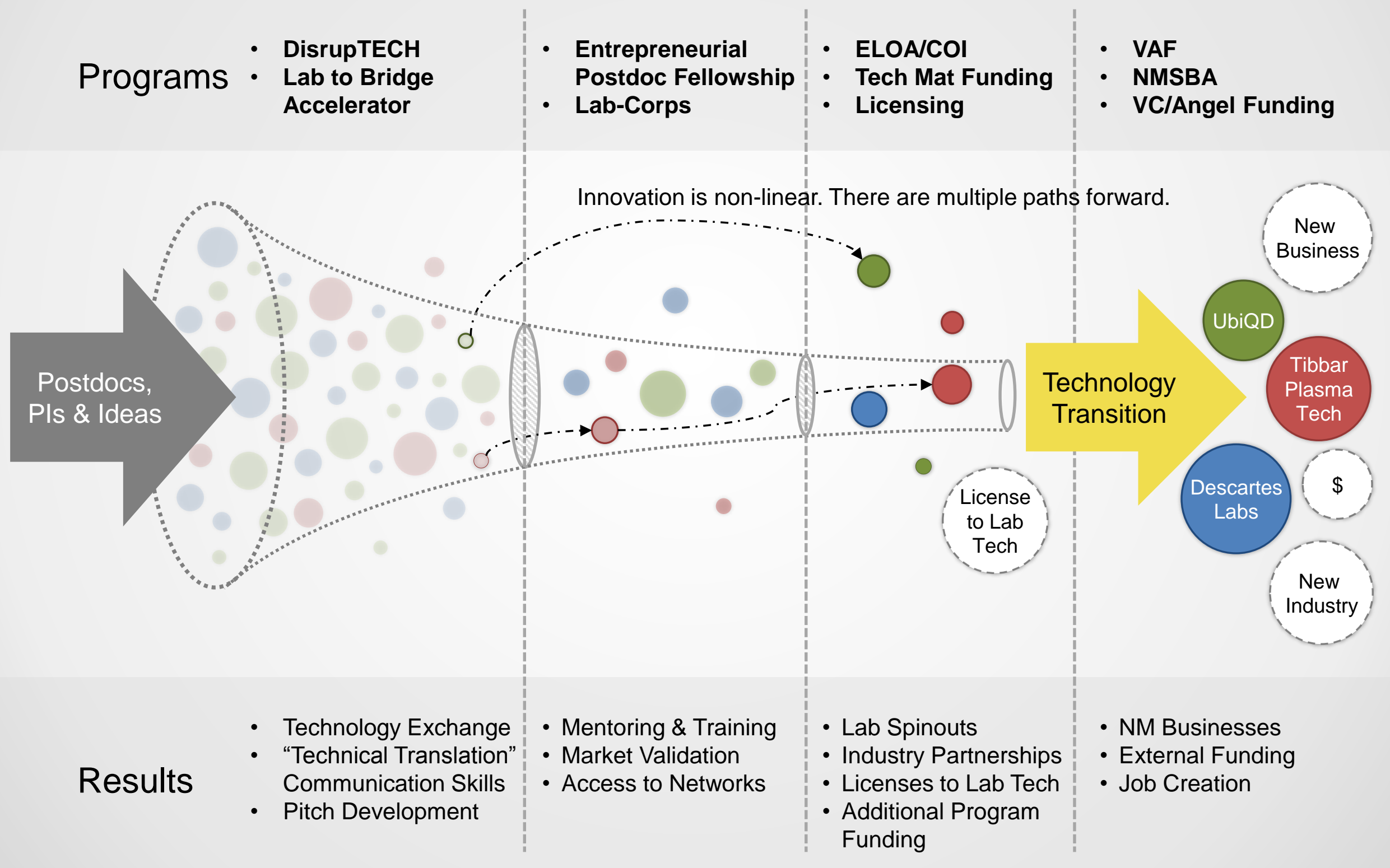




\section{Regional Economic Development}

- LANS Venture Acceleration Fund (VAF)

- Getting innovation to market faster

- New Mexico Small Business Assistance Program

- Solving New Mexico small business assistance challenges

- MBA internship

- Providing business data for entrepreneurs and inventors

- projectY cowork Los Alamos

- Collaborative open floor plan-programming, memberships, space rental 


\section{Los Alamos Launch Pad}

...a suite of programs for entrepreneurial learning

- DisrupTECH

- Coaching activities by industry experts that teaches refining their technical story and honing their pitch

- Lab Accelerator

- Collaborative lab effort to move technologies to market; 3-day UC Davis National Lab entrepreneurial training (with Sandia National Lab); national pitch competition

- UC/Los Alamos Entrepreneurial Postdoctoral Fellowship

- Pilot culminating in 6-month fellowship aimed at creating a new business in NNM

- Lab Corps

- Entrepreneurial education for national lab researchers, connects them to potential customers and industrial partners. Intensive 6 to 8 week program with follow-on coaching

Moving technology toward innovation and accelerating new businesses and VC investment 


\section{Northern New Mexico Innovation Ecosystem}

Porous boundary with ebb and flow of personnel and technology between LANL and the greater NNM Community, benefitted by a strong tech hub and entrepreneurial education institutions

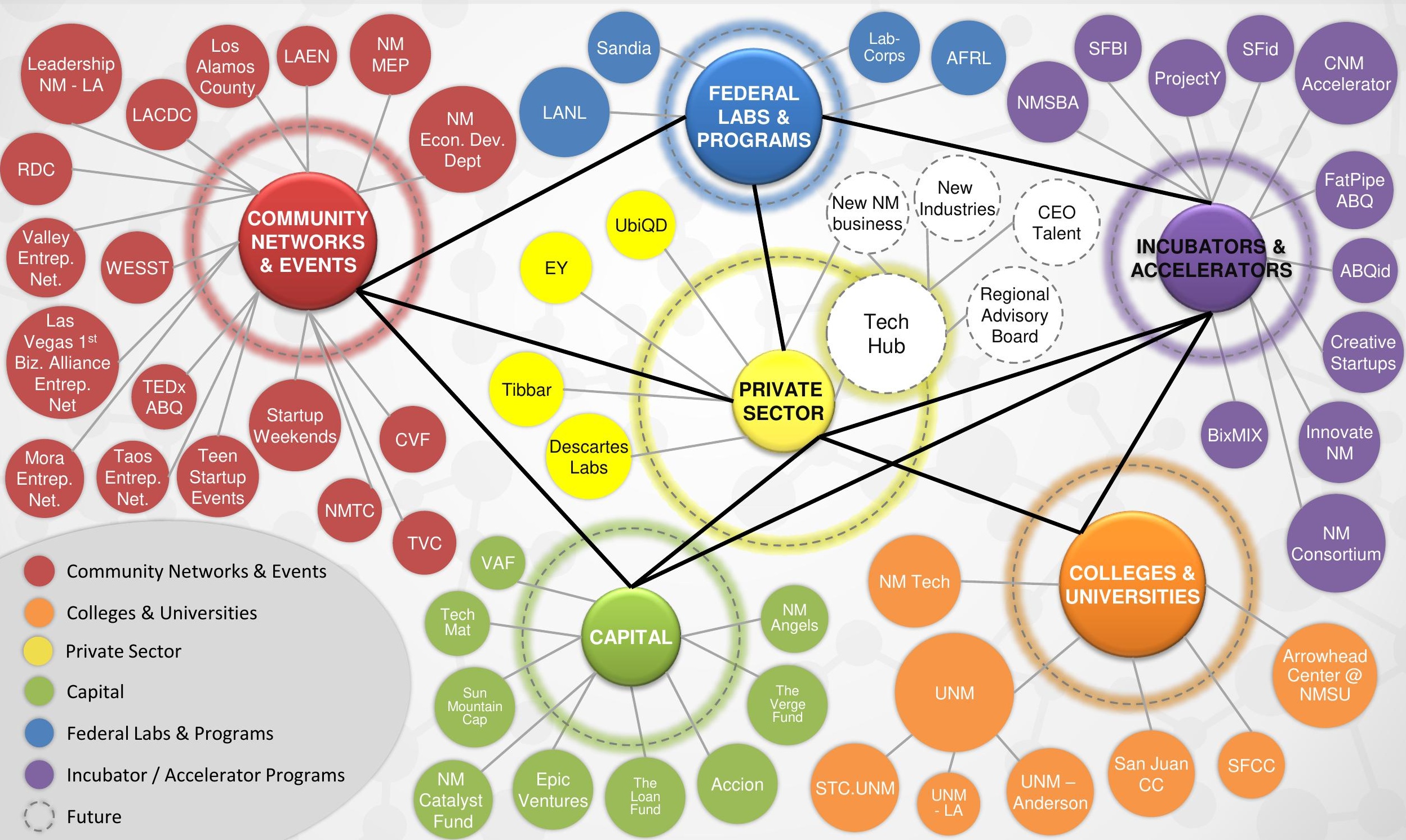




\section{Seeding Regional Activities}

\section{Power of the Network}

Farmington

San Juan Community College NM Manufacturing Extension Partnership

\section{Española} Regional Development Corp. Valley Entrepreneurial Network Teen Startup Events

\section{Los Alamos}

Leadership New Mexico - Los Alamos Nation Los Alamos Science Fest - LACDC Los Alamos County Los Alamos Entrepreneur's Network New Mexico Consortium Teen Startup Events

\section{Santa Fe} BizMIX Challenge - City of Santa Fe Santa Fe Business Incubator Santa Fe Community College Startup Weekend Events New Mexico Economic Development Department (Statewide)

\section{Socorro} New Mexico Tech

\section{Las Cruces}

Arrowhead Center at NMSU Startup Weekend Events

\section{Colorado}

National Renewable Energy Laboratory

Telluride Venture Accelerator

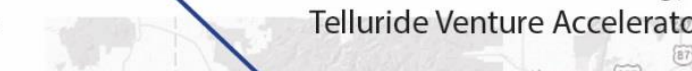

Taos

Taos Entrepreneurial Network Teen Startup Events

Mora Mora Entrepreneurial Network Las Vegas

Las Vegas First Business Alliance Entrepreneurial Network Albuquerque ABQid

Coronado Ventures Forum

New Mexico Angels

New Mexico Bio Association New Mexico Manufacturing Extension Partnership New Mexico Tech Council Technology Ventures Corp. TEDXABQ

The BioScience Center

UNM - Anderson School of Mgmt. Sandia National Laboratories

Teen Startup Events Startup Weekend Events STC.UNM WESST (Statewide)

Roswell

New Mexico Manufacturing Extension Partnership 